International Journal of Pure and Applied Mathematics

Volume 105 No. 4 2015, 811-822

ISSN: 1311-8080 (printed version); ISSN: 1314-3395 (on-line version)

url: http://www.ijpam.eu

doi: http://dx.doi.org/10.12732/ijpam.v105i4.20

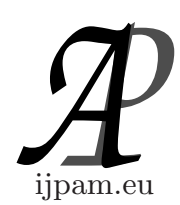

\title{
TRIPLE FIXED POINT THEOREMS FOR MAPPINGS SATISFYING WEAK CONTRACTIONS UNDER F-INVARIANT SET
}

\author{
Thokchom Chhatrajit Singh ${ }^{1 \S}$, Yumanm Rohen ${ }^{2}$ \\ ${ }^{1,2}$ Department of Basic Sciences and Humanities \\ NIT Manipur \\ Imphal, 795001, INDIA
}

\begin{abstract}
The aim of this paper to extend the results of the coupled fixed point theorems of Poom et. al. (2012) to triple fixed point theorems by weaking the concept of the mixed monotone property. The main results extend and unify the results of Poom et. al. and many results of the triple fixed point theorems.
\end{abstract}

AMS Subject Classification: 47H10, 54H25

Key Words: mixed monotone property, F-invariant set, cone metric space, fixed point theorems

\section{Introduction}

Bhaskar and Lakshmikanthan [1] proved the existence of a new fixed point theorem for a mixed monotone mapping in a metric space with the help of partial order, using a weak contractibity type of assumption. They named this new type of fixed point as coupled fixed point. Since then this new concept is extended and used in various directions. This concept is extended to tripled fixed point by Berinde and Borcut [2]. They obtained the existence and uniqueness

Received: October 13, 2015

(C) 2015 Academic Publications, Ltd.

${ }^{\S}$ Correspondence author url: www.acadpubl.eu 
theorems for contractive mappings in partially ordered complete metric spaces. This concept is extended to quadrupled fixed point by Karapinar [3]. In recent years many authors established various coupled fixed point theorems in cone metric space $[8,9,10,11,12,13,14,15,16,17,18,19]$ and references there in).

On the other hand, The concept of cone metric space introduced by Huang and Zang [4] in 2007 as generalizations of metric space. They generalised metric space by replacing the set of real numbers with an ordering Banach space. In recent years some authors established various triple fixed point theorems in cone metric space $[6,7]$ and references there in.

This paper extends the results of the coupled fixed point theorems of Poom et. al. (2012) to triple fixed point theorems by weaking the concept of the mixed monotone property. The main results extend and unify the results of Poom et. al. and many results of the triple fixed point theorems.

\section{Preliminary Notes}

Given a cone $P \subset E$ we define the partial ordering $\leq$ with respect to $P$ by $x \leq y$ if and only if $y-x \in P$. We write $x<y$ to denote that $x \leq y$ but $x \neq y$, while $x<<y$ will stand for $y-x \in$ int.P(interior of $P$ ).

There are two kinds of cone. They are normal cone and non-normal cones. A cone $P \subset E$ is normal if there is a number $K>0$ such that for all $x, y \in P$, $0 \leq x \leq y \Rightarrow\|x\| \leq K\|y\|$. In other words if $x_{n} \leq y_{n} \leq z_{n}$ and $\lim _{n \rightarrow \infty} x_{n}=$ $\lim _{n \rightarrow \infty} z_{n}=x$ imply $\lim _{n \rightarrow \infty} y_{n}=x$. Also, a cone $P \subset E$ is regular if every increasing sequence which is bounded above is convergent.

The aim of the present work is to prove the existence and uniqueness of a common coupled fixed point theorem satisfying a generalised contractive condition. Some other results are also given in the form of corollaries.

We shave have following definitions.

Definition 2.1. [4] Let $X$ be a nonempty set. Suppose the mapping $d: X \times X \rightarrow E$ satisfies the following conditions:

1. $0<d(x, y)$ for all $x, y \in X$ and $d(x, y)=0$ iff $x=y$.

2. $d(x, y)=d(y, x)$ for all $x, y \in X$.

3. $d(x, y) \leq d(x, z)+d(z, y)$ for all $x, y, z \in X$.

Then $d$ is called a cone metric on $X$ and $(X, d)$ is called a cone metric space. 
Definition 2.2. [5] Let $(X, d)$ be a cone metric space and $F: X \times X \times X \rightarrow$ $X$ a given mapping. Let $\mathrm{M}$ be a non-empty subset of $X^{4}$. One say that $\mathrm{M}$ is the F-invariant subset of $X^{4}$ if and only if, for all $x, y, w \in X$, one has

$$
\begin{aligned}
1 .(x, y, z, w) \in M \Leftrightarrow & (w, z, y, x) \in M \\
2 .(x, y, z, w) \in M \Leftrightarrow & (F(x, y, z), F(z, y, x), F(y, z, w), \\
& F(w, z, y), F(z, w, x), F(x, w, z)) \in M .
\end{aligned}
$$

Example 2.3. $[5]$ Let $(X, d)$ be a cone metric space endowed with a partial order $\leq$. Let $F: X^{3} \rightarrow X$ be a mapping satisfying the mixed monofone property, for all $x, y \in X$, we have

$$
\begin{array}{r}
x_{1}, x_{2} \in X, x_{1} \leq x_{2} \quad \Rightarrow \quad F\left(x_{1}, y, z\right) \leq F\left(x_{2}, y, z\right) \\
y_{1}, y_{2} \in Y, y_{1} \leq y_{2} \quad \Rightarrow \quad F\left(x, y_{1}, z\right) \leq F\left(x, y_{2}, z\right) \\
z_{1}, z_{2} \in X, z_{1} \leq z_{2} \quad \Rightarrow \quad F\left(x, y, z_{1}\right) \leq F\left(x, y, z_{2}\right) .
\end{array}
$$

Define the subset $M \subseteq X^{4}$ by

$$
M=\{(a, b, c, d): c \leq a, b \leq d\} .
$$

Then, $\mathrm{M}$ is the F-invariant of $X^{4}$.

Definition 2.4. Let $(X, d)$ be a cone metric space and $F: X^{3} \rightarrow X$ a given mapping. Let $\mathrm{M}$ be a nonempty subset of $x^{6}$. One says that $\mathrm{M}$ is the F-invariant subset of $x^{6}$ if and only if for all $x, y, z,, u, v, w \in X$,

$$
\begin{gathered}
(x, y, z, u, v, w) \in M \Leftrightarrow(w, v, u, z, y, x) \in M \\
(x, y, z, u, v, w) \in \\
\Rightarrow(F(x, y, z), F(y, x, z), F(z, y, x), F(u, v, w), F(v, u, w), F(w, v, u)) \in M .
\end{gathered}
$$

\section{Main Results}

We have the following theorems.

Theorem 3.1. Let $(X, d)$ be a complete cone metric space. Let $q$ be a c-distance on $X, M$ a nonempty subset of $X^{6}$ and $F: X \rightarrow X$ a continuous function such that

$$
q\left(F(x, y, z), F\left(x^{*}, y^{*}, z^{*}\right)\right) \leq \frac{k}{4}\left(q\left(x, x^{*}\right)+q\left(y, y^{*}\right)+q\left(z, z^{*}\right)\right),
$$


for some $k \in[0,1]$ and $x, y, z, x^{*}, y^{*}, z^{*} \in X$ with $\left(x, y, z, x^{*}, y^{*}, z^{*}\right) \in M$ or $\left(x^{*}, y^{*}, z^{*}, x, y, z\right) \in M$.

If $M$ is an F-invariant and there exist $x_{0}, y_{0}, z_{0} \in X$ such that

$$
\left(F\left(x_{0}, y_{0}, z_{0}\right), F\left(y_{0}, x_{0}, z_{0}\right), F\left(z_{0}, y_{0}, x_{0}\right), x_{0}, y_{0}, z_{0}\right) \in M
$$

then $F$ has a complete fixed point $(u, v, w)$.

Proof. As $F(X \times X \times X) \subseteq X$, we can construct sequences $\left\{x_{n}\right\},\left\{y_{n}\right\}$ and $\left\{z_{n}\right\}$ in $\mathrm{X}$ such that

$$
\begin{aligned}
& x_{n}=F\left(x_{n-1}, y_{n-1}, z_{n-1}\right) \\
& y_{n}=F\left(y_{n-1}, x_{n-1}, y_{n-1}\right)
\end{aligned}
$$

and

$$
z_{n}=F\left(z_{n-1}, y_{n-1}, x_{n-1}\right), \forall n \in N
$$

Since $\left(F\left(x_{0}, y_{0}, z_{0}\right), F\left(y_{0}, x_{0}, z_{0}\right), F\left(z_{0}, y_{0}, x_{0}\right), x_{0}, y_{0}, z_{0}\right) \in M$ and $\mathrm{M}$ is an Finvariant set, we get

$$
\begin{array}{r}
\left(F\left(x_{1}, y_{1}, z_{1}\right), F\left(y_{1}, x_{1}, y_{1}\right), F\left(z_{1}, y_{1}, x_{1}\right), F\left(x_{0}, y_{0}, z_{0}\right), F\left(y_{0}, x_{0}, z_{0}\right),\right. \\
\left.F\left(z_{0}, y_{0}, x_{0}\right)\right)=\left(x_{2}, y_{2}, z_{2}, x_{1}, y_{1}, z_{1}\right) \in M .
\end{array}
$$

Again using the fact that $\mathrm{M}$ is an F-invariant set, we have

$$
\begin{array}{r}
\left(F\left(x_{2}, y_{2}, z_{2}\right), F\left(y_{2}, x_{2}, z_{2}\right), F\left(z_{2}, y_{2}, x_{2}\right), F\left(x_{1}, y_{1}, z_{1}\right), F\left(y_{1}, x_{1}, z_{1}\right),\right. \\
\left.F\left(z_{1}, y_{1}, x_{1}\right)\right)=\left(x_{3}, y_{3}, z_{3}, x_{2}, y_{2}, z_{2}\right) \in M .
\end{array}
$$

By repeating the argument to the above, we get

$$
\begin{aligned}
& \left(F\left(x_{n-1}, y_{n-1}, z_{n-1}\right), F\left(y_{n-1}, x_{n-1}, z_{n-1}\right)\right. \\
& \left.\left.F\left(z_{n-1}, y_{n-1}, x_{n-1}\right), x_{n-1}, y_{n-1}, z_{n-1}\right)\right)=\left(x_{n}, y_{n}, z_{n}, x_{n-1}, y_{n-1}, z_{n-1}\right) \in M
\end{aligned}
$$

Then

$$
\begin{aligned}
q\left(x_{n}, x_{n+1}\right) & =q\left(\left(F\left(x_{n-1}, y_{n-1}, z_{n-1}\right)\right),\left(F\left(x_{n}, y_{n}, z_{n}\right)\right.\right. \\
& \leq \frac{k}{3}\left(q\left(x_{n-1}, x_{n}\right)+q\left(y_{n-1}, y_{n}\right)+q\left(z_{n-1}, z_{n}\right)\right) \\
q\left(x_{n+1}, x_{n}\right) & =q\left(\left(F\left(x_{n}, y_{n}, z_{n}\right)\right),\left(F\left(x_{n-1}, y_{n-1}, z_{n-1}\right)\right.\right.
\end{aligned}
$$




$$
\begin{aligned}
\leq & \frac{k}{3}\left(q\left(x_{n}, x_{n-1}\right)+q\left(y_{n}, y_{n-1}\right)+q\left(z_{n}, z_{n-1}\right)\right) \\
q\left(x_{n}, x_{n+1}\right)+q\left(x_{n+1}, x_{n}\right) \leq & \frac{k}{3}\left[\left(q\left(x_{n-1}, x_{n}\right)+q\left(y_{n-1}, y_{n}\right)+q\left(z_{n-1}, z_{n}\right)\right.\right. \\
& \left.\left.+q\left(x n, x_{n-1}\right)+q\left(y_{n}, y_{n-1}\right)+q\left(z_{n}, z_{n-1}\right)\right)\right]
\end{aligned}
$$

Since $\left(x_{n}, y_{n}, z_{n}, x_{n-1}, y_{n-1}, z_{n-1}\right) \in M$ for all $n \in M$ is an F-invariant set, we get $\left(z_{n-1}, y_{n-1}, x_{n-1}, x_{n}, y_{n}, z_{n}\right) \in M$ for all $n \in N$. We have

$$
\begin{aligned}
q\left(y_{n}, y_{n+1}\right) & =q\left(\left(F\left(y_{n-1}, x_{n-1}, y_{n-1}\right)\right),\left(F\left(y_{n}, x_{n}, y_{n}\right)\right.\right. \\
& \leq \frac{k}{2}\left(q\left(y_{n-1}, y_{n}\right)+q\left(x_{n-1}, x_{n}\right)+q\left(z_{n-1}, z_{n}\right)\right)
\end{aligned}
$$

and

$$
\begin{aligned}
q\left(y_{n+1}, y_{n}\right) & =q\left(\left(F\left(y_{n}, x_{n}, y_{n}\right)\right),\left(F\left(y_{n-1}, x_{n-1}, y_{n-1}\right)\right.\right. \\
& \leq \frac{k}{3}\left(q\left(y_{n}, y_{n-1}\right)+q\left(x_{n}, x_{n-1}\right)+q\left(z_{n}, z_{n-1}\right)\right) \\
& \leq \frac{k}{3}\left(2 q\left(y_{n}, y_{n-1}\right)+q\left(x_{n}, x_{n-1}\right)\right) .
\end{aligned}
$$

Adding (3) and (4):

$$
\begin{aligned}
q\left(y_{n}, y_{n+1}\right)+ & q\left(y_{n+1}, y_{n}\right) \\
& \leq \frac{k}{3}\left[\left(q\left(y_{n-1}, x_{n}\right)+2 q\left(y_{n-1}, y_{n}\right)+q\left(x_{n}, x_{n-1}\right)+2 q\left(y n, y_{n-1}\right)\right]\right.
\end{aligned}
$$

Also, we have

$$
\begin{aligned}
q\left(z_{n}, z_{n+1}\right)= & q\left(\left(F\left(z_{n-1}, y_{n-1}, x_{n-1}\right)\right),\left(F\left(z_{n}, y_{n}, x_{n}\right)\right.\right. \\
\leq & \frac{k}{3}\left(q\left(z_{n-1}, z_{n}\right)+q\left(y_{n-1}, y_{n}\right)+q\left(x_{n-1}, x_{n}\right)\right), \\
q\left(z_{n+1}, z_{n}\right)= & q\left(\left(F\left(z_{n}, y_{n}, x_{n}\right)\right),\left(F\left(z_{n-1}, y_{n-1}, x_{n-1}\right)\right.\right. \\
\leq & \frac{k}{3}\left(q\left(z_{n}, z_{n-1}\right)+q\left(y_{n}, y_{n-1}\right)+q\left(x_{n}, x_{n-1}\right)\right), \\
q\left(z_{n}, z_{n+1}\right)+q\left(z_{n+1}, z_{n}\right) \leq & \frac{k}{3}\left[q\left(z_{n-1}, z_{n}\right)+q\left(y_{n-1}, y_{n}\right)+q\left(x_{n-1}, x_{n}\right)\right. \\
& \left.+q\left(z_{n}, z_{n-1}\right)+q\left(y_{n}, y_{n-1}\right)+q\left(x_{n}, x_{n-1}\right)\right] .
\end{aligned}
$$

Adding the above equalities, we receive 


$$
\begin{gathered}
q\left(x_{n}, x_{n+1}\right)+q\left(x_{n+1}, x_{n}\right)+q\left(y_{n}, y_{n+1}\right)+q\left(y_{n+1}, y_{n}\right)+q\left(z_{n}, z_{n+1}\right)+q\left(z_{n+1}, z_{n}\right) \leq \\
k\left(q\left(x_{n-1}, x_{n}\right)+q\left(y_{n-1}, y_{n}\right)+q\left(z_{n-1}, z_{n}\right)+q\left(x_{n}, x_{n-1}\right)+q\left(y_{n}, y_{n-1}\right)+q\left(z_{n}, z_{n-1}\right)\right) .
\end{gathered}
$$

If we repeat the above process for $n$-times, then we get

$$
\begin{gathered}
q\left(x_{n}, x_{n+1}\right)+q\left(x_{n+1}, x_{n}\right)+q\left(y_{n}, y_{n+1}\right)+q\left(y_{n+1}, y_{n}\right)+q\left(z_{n}, z_{n+1}\right)+q\left(z_{n+1}, z_{n}\right) \\
\leq k^{n}\left(q\left(x_{1}, x_{0}\right)+q\left(y_{1}, y_{0}\right)+q\left(z_{1}, z_{0}\right)+q\left(x_{0}, x_{1}\right)+q\left(y_{0}, y_{1}\right)+q\left(z_{0}, z_{1}\right)\right) .
\end{gathered}
$$

Therefore

$$
\begin{aligned}
& q\left(x_{n}, x_{n+1}\right) \leq k^{n}\left(q\left(x_{1}, x_{0}\right)+q\left(y_{1}, y_{0}\right)+q\left(z_{1}, z_{0}\right)+q\left(x_{0}, x_{1}\right)+q\left(y_{0}, y_{1}\right)\right. \\
& \left.+q\left(z_{0}, z_{1}\right)\right) \text {, } \\
& q\left(y_{n}, y_{n+1}\right) \leq k^{n}\left(q\left(x_{1}, x_{0}\right)+q\left(y_{1}, y_{0}\right)+q\left(z_{1}, z_{0}\right)+q\left(x_{0}, x_{1}\right)+q\left(y_{0}, y_{1}\right)\right. \\
& \left.+q\left(z_{0}, z_{1}\right)\right) \text {, }
\end{aligned}
$$

and

$q\left(z_{n}, z_{n+1}\right) \leq k^{n}\left(q\left(x_{1}, x_{0}\right)+q\left(y_{1}, y_{0}\right)+q\left(z_{1}, z_{0}\right)+q\left(x_{0}, x_{1}\right)+q\left(y_{0}, y_{1}\right)+q\left(z_{0}, z_{1}\right)\right)$.

Let $m, n \in N$ with $m>n$. Since

$$
\begin{aligned}
q\left(x_{n}, x_{m}\right) & \leq \sum_{i=n}^{m-1} q\left(x_{i}, x_{i+1}\right) \\
q\left(y_{n}, y_{m}\right) & \leq \sum_{i=n}^{m-1} q\left(y_{i}, y_{i+1}\right)
\end{aligned}
$$

and

$$
q\left(z_{n}, z_{m}\right) \leq \sum_{i=n}^{m-1} q\left(z_{i}, z_{i+1}\right)
$$

we obtain $(0 \leq k<1)$ :

$$
\begin{aligned}
q\left(x_{n}, x_{n+1}\right) \leq & \frac{k^{n}}{1-k}\left(q\left(x_{1}, x_{0}\right)+q\left(y_{1}, y_{0}\right)+q\left(z_{1}, z_{0}\right)+q\left(x_{0}, x_{1}\right)+q\left(y_{0}, y_{1}\right)\right. \\
& \left.+q\left(z_{0}, z_{1}\right)\right), \\
q\left(y_{n}, y_{n+1}\right) \leq & \frac{k^{n}}{1-k}\left(q\left(x_{1}, x_{0}\right)+q\left(y_{1}, y_{0}\right)+q\left(z_{1}, z_{0}\right)+q\left(x_{0}, x_{1}\right)+q\left(y_{0}, y_{1}\right)\right. \\
& \left.+q\left(z_{0}, z_{1}\right)\right),
\end{aligned}
$$




$$
\begin{aligned}
q\left(z_{n}, z_{n+1}\right) \leq & \frac{k^{n}}{1-k}\left(q\left(x_{1}, x_{0}\right)+q\left(y_{1}, y_{0}\right)+q\left(z_{1}, z_{0}\right)+q\left(x_{0}, x_{1}\right)+q\left(y_{0}, y_{1}\right)\right. \\
& \left.+q\left(z_{0}, z_{1}\right)\right)
\end{aligned}
$$

Using the lemma, we have $\left\{x_{n}\right\},\left\{y_{n}\right\}$ and $\left\{z_{n}\right\}$ are chauchy sequences in $(X, d)$. By the completeness of $\mathrm{X}$. we get $x_{n} \rightarrow u, y_{n} \rightarrow v$ and $z_{n} \rightarrow w$ for $u, v, w \in X$. Since $\mathrm{F}$ is continuous, taking $n \rightarrow \infty$, we get

$\lim _{n \rightarrow \infty} x_{n+1}=\lim _{n \rightarrow \infty} F\left(x_{n}, y_{n}, z_{n}\right)=F\left(\lim _{n \rightarrow \infty} x_{n}, \lim _{n \rightarrow \infty} y_{n}, \lim _{n \rightarrow \infty} z_{n}\right)=F(u, v, w)$,

$\lim _{n \rightarrow \infty} y_{n+1}=\lim _{n \rightarrow \infty} F\left(y_{n}, x_{n}, y_{n}\right)=F\left(\lim _{n \rightarrow \infty} y_{n}, \lim _{n \rightarrow \infty} x_{n}, \lim _{n \rightarrow \infty} y_{n}\right)=F(v, u, v)$,

$\lim _{n \rightarrow \infty} z_{n+1}=\lim _{n \rightarrow \infty} F\left(z_{n}, y_{n}, x_{n}\right)=F\left(\lim _{n \rightarrow \infty} z_{n}, \lim _{n \rightarrow \infty} y_{n}, \lim _{n \rightarrow \infty} x_{n}\right)=F(w, v, u)$.

By the uniqueness of the limits, we get $u=F(u, v, w), v=F(v, u, v)$ and $w=F(w, v, u)$. Therefore, $(\mathrm{u}, \mathrm{v}, \mathrm{w})$ is a triple fixed point of $\mathrm{F}$.

Finally, we assume that $(u, v, w, u, v, w) \in M$ and so $(w, v, u, w, v, u) \in M$. We have

$$
\begin{aligned}
q(u, u) & =q(F(u, v, w), F(u, v, w)) \\
& \leq \frac{k}{3}(q(u, u)+q(v, v)+q(w, w)) \\
q(v, v) & =q(F(v, u, v), F(v, u, v)) \\
& \leq \frac{k}{3}(q(v, v)+q(u, u)+q(v, v)),
\end{aligned}
$$

and

$$
\begin{aligned}
q(w, w) & =q(F(w, v, u), F(w, v, u)) \\
& \leq \frac{k}{3}(q(u, u)+q(v, v)+q(w, w)) .
\end{aligned}
$$

Therefore

$$
q(u, u)+q(v, v)+q(w, w) \leq k(q(u, v)+q(v, v)+q(w, w)) .
$$

Since $0 \leq k<1$, we conclude that $q(u, u)+q(v, v)+q(w, w)=0$ and hence $q(u, u)=0, q(v, v)=0$ and $q(w, w)=0$. 
Theorem 3.2. Let $(X, d)$ be a complete cone metric space. Let $q$ be a c-distance on $X, M$ a subset of $X^{6}$ and $F: X \times X \rightarrow X$ a function such that

$$
q\left(F(x, y, z), F\left(x^{*}, y^{*}, z^{*}\right)\right) \leq \frac{k}{6}\left(q\left(x, x^{*}\right)+q\left(y, y^{*}\right)+q\left(z, z^{*}\right)\right)
$$

for some $k \in[0,1]$ and for all $x, y, z, x^{*}, y^{*}, z^{*} \in X$ with $\left(x, y, z, x^{*}, y^{*}, z^{*}\right) \in M$ or $\left(x^{*}, y^{*}, z^{*}, x, y, z\right) \in M$.

Also, suppose that:

1. There exists $x_{0}, y_{0}, z_{0} \in X$ and such that

$$
\left(F\left(x_{0}, y_{0}, z_{0}\right), F\left(x_{0}, y_{0}, z_{0}\right), F\left(x_{0}, y_{0}, z_{0}\right), x_{0}, y_{0}, z_{0}\right) \in M \text {. }
$$

2. If two sequences $\left\{x_{n}\right\},\left\{y_{n}\right\}$ and $\left\{z_{n}\right\}$ are such that

$$
\left(x_{n+1}, y_{n+1}, z_{n+1}, x_{n}, y_{n}, z_{n}\right) \in M,
$$

for all $n \in N$ and $\left\{x_{n}\right\} \rightarrow x,\left\{y_{n}\right\} \rightarrow y$ and $\left\{z_{n}\right\} \rightarrow z$, then

$$
\left(x_{n}, y_{n}, z_{n}, x, y, z\right) \in M
$$

for all $n \in N$.

If $M$ is an $F$-invariant set, then $F$ has a triple fixed point.

Proof. As in the proof of theorem 3.3, we can construct two cauchy sequences $\left\{x_{n}\right\},\left\{y_{n}\right\}$ and $\left\{z_{n}\right\}$ in $\mathrm{X}$ such that $\left(x_{n}, y_{n}, z_{n}, x_{n-1}, y_{n-1}, z_{n-1}\right) \in M$ for all $n \in N$. Moreover, we have $\left\{x_{n}\right\}$ converges to a point $u \in X,\left\{y_{n}\right\}$ converges to a point $v \in X$ and $\left\{z_{n}\right\}$ converges to a point $w \in X$.

$$
\begin{aligned}
q\left(x_{n}, x_{m}\right) \leq & \frac{k^{n}}{1-k}\left(q\left(x_{1}, x_{0}\right)+q\left(y_{1}, y_{0}\right)+q\left(z_{1}, z_{0}\right)+q\left(x_{0}, x_{1}\right)+q\left(y_{0}, y_{1}\right)\right. \\
& \left.+q\left(z_{0}, z_{1}\right)\right), \\
q\left(y_{n}, y_{m}\right) \leq & \frac{k^{n}}{1-k}\left(q\left(x_{1}, x_{0}\right)+q\left(y_{1}, y_{0}\right)+q\left(z_{1}, z_{0}\right)+q\left(x_{0}, x_{1}\right)+q\left(y_{0}, y_{1}\right)\right. \\
& \left.+q\left(z_{0}, z_{1}\right)\right),
\end{aligned}
$$

and

$$
\begin{aligned}
q\left(z_{n}, z_{m}\right) \leq & \frac{k^{n}}{1-k}\left(q\left(x_{1}, x_{0}\right)+q\left(y_{1}, y_{0}\right)+q\left(z_{1}, z_{0}\right)+q\left(x_{0}, x_{1}\right)+q\left(y_{0}, y_{1}\right)\right. \\
& \left.+q\left(z_{0}, z_{1}\right)\right)
\end{aligned}
$$


for each $m>n \geq 1$. Hence, we have

$$
\begin{aligned}
q\left(x_{n}, u\right) \leq & \frac{k^{n}}{1-k}\left(q\left(x_{1}, x_{0}\right)+q\left(y_{1}, y_{0}\right)+q\left(z_{1}, z_{0}\right)+q\left(x_{0}, x_{1}\right)+q\left(y_{0}, y_{1}\right)\right. \\
& \left.+q\left(z_{0}, z_{1}\right)\right) \\
q\left(y_{n}, v\right) \leq & \frac{k^{n}}{1-k}\left(q\left(x_{1}, x_{0}\right)+q\left(y_{1}, y_{0}\right)+q\left(z_{1}, z_{0}\right)+q\left(x_{0}, x_{1}\right)+q\left(y_{0}, y_{1}\right)\right. \\
& \left.+q\left(z_{0}, z_{1}\right)\right)
\end{aligned}
$$

and

$$
\begin{aligned}
q\left(z_{n}, w\right) \leq & \frac{k^{n}}{1-k}\left(q\left(x_{1}, x_{0}\right)+q\left(y_{1}, y_{0}\right)+q\left(z_{1}, z_{0}\right)+q\left(x_{0}, x_{1}\right)+q\left(y_{0}, y_{1}\right)\right. \\
& \left.+q\left(z_{0}, z_{1}\right)\right) .
\end{aligned}
$$

Therefore

$$
\begin{aligned}
q\left(x_{n}, u\right)+q\left(y_{n}, v\right)+q\left(z_{n}, w\right) \leq & \frac{3 k^{n}}{1-k}\left(q\left(x_{1}, x_{0}\right)+q\left(y_{1}, y_{0}\right)+q\left(z_{1}, z_{0}\right)\right. \\
& \left.+q\left(x_{0}, x_{1}\right)+q\left(y_{0}, y_{1}\right)+q\left(z_{0}, z_{1}\right)\right) .
\end{aligned}
$$

By assumptions, we have $\left(u, v, w, x_{n-1}, y_{n-1}, z_{n-1}\right) \in M$ and

$$
\left(y_{n-1}, x_{n-1}, y_{n-1}, v, u, v\right) \in M \text { and }\left(z_{n-1}, y_{n-1}, x_{n-1}, v, u, v\right) \in M .
$$

We have

$$
\begin{aligned}
q\left(x_{n}, F(u, v, w)\right) & =q\left(F\left(x_{n-1}, y_{n-1}, z_{n-1}\right), F(u, v, w)\right) \\
& \leq \frac{k}{6}\left(q\left(x_{n-1}, u\right)+q\left(y_{n-1}, v\right)+q\left(z_{n-1}, w\right)\right) . \\
q\left(y_{n}, F(v, u, v)\right) & =q\left(F\left(y_{n-1}, x_{n-1}, y_{n-1}\right), F(v, u, v)\right) \\
& \leq \frac{k}{6}\left(q\left(y_{n-1}, v\right)+q\left(x_{n-1}, u\right)+q\left(y_{n-1}, v\right)\right) .
\end{aligned}
$$

and

$$
\begin{aligned}
q\left(z_{n}, F(w, v, u)\right) & =q\left(F\left(z_{n-1}, y_{n-1}, x_{n-1}\right), F(w, v, u)\right) \\
& \leq \frac{k}{6}\left(q\left(z_{n-1}, w\right)+q\left(y_{n-1}, v\right)+q\left(x_{n-1}, u\right)\right)
\end{aligned}
$$

Moreover

$$
q\left(x_{n}, F(u, v, w)\right)+q\left(y_{n}, F(v, u, v)\right)+q\left(z_{n}, F(v, u, v)\right) \leq
$$




$$
\begin{aligned}
& \frac{k}{3} \cdot \frac{3 k^{n-1}}{1-k}\left(q\left(x_{1}, x_{0}\right)+q\left(y_{1}, y_{0}\right)\right. \\
& +q\left(z_{1}, z_{0}\right)+q\left(x_{0}, x_{1}\right)+q\left(y_{0}, y_{1}\right)+q\left(z_{0}, z_{1}\right) \\
= & \frac{k^{n}}{1-k}\left(q\left(x_{1}, x_{0}\right)+q\left(y_{1}, y_{0}\right)\right. \\
& +q\left(z_{1}, z_{0}\right)+q\left(x_{0}, x_{1}\right)+q\left(y_{0}, y_{1}\right)+q\left(z_{0}, z_{1}\right) .
\end{aligned}
$$

Therefore, we have

$$
\begin{aligned}
q\left(x_{n}, F(u, v, w)\right) \leq & \frac{k^{n}}{1-k}\left(q\left(x_{1}, x_{0}\right)+q\left(y_{1}, y_{0}\right)\right. \\
& +q\left(z_{1}, z_{0}\right)+q\left(x_{0}, x_{1}\right)+q\left(y_{0}, y_{1}\right)+q\left(z_{0}, z_{1}\right) \\
q\left(y_{n}, F(v, u, v)\right) \leq & \frac{k^{n}}{1-k}\left(q\left(x_{1}, x_{0}\right)+q\left(y_{1}, y_{0}\right)\right. \\
& +q\left(z_{1}, z_{0}\right)+q\left(x_{0}, x_{1}\right)+q\left(y_{0}, y_{1}\right)+q\left(z_{0}, z_{1}\right) .
\end{aligned}
$$

and

$$
\begin{aligned}
q\left(z_{n}, F(v, u, v)\right) \leq & \frac{k^{n}}{1-k}\left(q\left(x_{1}, x_{0}\right)+q\left(y_{1}, y_{0}\right)\right. \\
& +q\left(z_{1}, z_{0}\right)+q\left(x_{0}, x_{1}\right)+q\left(y_{0}, y_{1}\right)+q\left(z_{0}, z_{1}\right)
\end{aligned}
$$

This implies that $u=F(u, v, w), u=F(v, u, v)$ and $w=F(w, u, w)$.

\section{References}

[1] T.G Bhaskar and V. Lakshmikanthan, Fixed point theorems in partially oredered metric spaces and applications, Nonlinear Analysis:TMA, 65 (2006) 1379-1393.

[2] Berinde, V., Borcut M., Tripled fixed point theorems for contractive type mappings in partially ordered metric spaces, Nonlinear Anal., 74 (2011) 4889-4897.

[3] E. Karapinar, Couple Fixed Point on Cone Metric Space, Stud. Univ. Babes-Bolyai Math., 58 (2013) 75-85.

[4] L.G.Huang and X. Zhang, Cone metric spaces and fixed point theorems of contractive mapping, J. Math. Anal. Appl., 332 (2007) 1468-1476. 
[5] W. Sintunavarat, Y.J. Cho, P. Kumam, Coupled Fixed Point Theorems for Weak Contraction Mappings under F-Invariant Set, Abstarct and Applied Analysis, doi: 10.1155/2012/324874.

[6] H. Aydi and E. Karapinar, Triple Fixed Point in Oredered Metric Space, Bulletin of Mathematical Analysis and Applications, 4 (2012) 197-207.

[7] T.C. Singh and Y. R. Singh., Triple Fixed Points Theorems on Cone Banach Space, it Journal of Global Research in Mathematical Archives, 2 (2014) 43-49.

[8] M. Abbas, M. Ali Khan, S. Radenovic, Common coupled fixed point theorems in cone metric spaces for w-compatible mappings, Applied Mathematics and Computation, 217 (2010) 195-202.

[9] E. Karapinar, Coupled Fixed Point on Cone Metric Spaces, Gazi University Journal of Science, 1 (2011) 51-58.

[10] W. Long, B.E Rhoades and M. Rajovic, Copled Coincidence points for two mappings in metric spaces and cone metric spaces, Fixed Point Theory and Applications, 2012 (2012) 1-9.

[11] Z. Kadelburg and S. Radenovic, Coupled Fixed point results under TVScone metric and w-cone-distance, Advances in Fixed point Theory, 2 (2012) $29-46$.

[12] R. Batra and S.Vashistha, Coupled coincidence point theorems for nonlinear contractions under $(F, g)$-invariant set in cone metric spaces, J. Nonlinear Sci. Appl., 6(2013) 86-96.

[13] R. Batra, S. Vashistha and R. Kumar, Coupled coincidence point theorems for mappings without mixed monotone property under c-distance in cone metric spaces, J. Nonlinear Sci. Appl., 7 (2014) 345-358.

[14] E. Karapinar and D. Turkoglu, Best Approximations Theorems for a couple in Cone Banach Space, Fixed Point Theory and Applications, 2010 doi: $10.1155 / 2010 / 784578$.

[15] W. Shantanawi, Some Common Coupled Fixed Point results in cone metric spaces, Int. Journal of Math. Analysis, 4 (2010) 2381-2388.

[16] A. Aghajani, M. Abbas and E. P. Kallehbasti, Coupled fixed point theorems in partially ordered metric spaces and application, Math. Commun., 17 (2012) 497-509. 
[17] S. Sedghi, N. Shobkolaei,J. R. Roshan and W. Shatanawi, Coupled fixed point theorems $G_{b}$-Metric Space, MATEMATIQKI VESNIK, 2 (2014) 190201.

[18] Jay G. Mehta and M. L. Joshi, On Coupled Fixed Point Theorem in Partially Ordered Complete Metric Space, Int. J. Pure Appl. Sci. Technol., 1 (2010) 87-92.

[19] N. Malhotra and B.Bansal, Some common coupled fixed point theorems for generalized contraction in b-metric space, J. Nonlinear Sci. Appl., 8 (2015) 8-16. 